\title{
Efficacy of fish body oil in the treatment of rheumatoid arthritis: a systematic review
}

\author{
De Silva PV \\ Department of Community Medicine, Faculty of Medicine, University of Ruhuna, Sri Lanka
}

Correspondence: Dr.P.V.de Silva (pvdesilva@med.ruh.ac.lk)

\begin{abstract}
Introduction: Rheumatoid arthritis (RA) is a chronic autoimmune disease with intermittent exacerbations and remissions. A large number of different therapies including alternative and complementary medicines have been used to treat RA without much evidence about their success. In recent times, fish body oil has attracted popularity among the physicians in Sri Lanka. The objective of this paper is to produce evidence on efficacy of fish oil in the treatment of RA.

Materials and Methods: Publications to include in the present review were retrieved using a computerized search of seven independent databases. The references of all selected relevant articles were manually searched to obtain additional relevant articles. Only the RCTs published in English language were selected.

Results: Efficacy of fish body oil in the management of RA has been tested in $12 \mathrm{RCTs}$ with sample size ranging from 16 to 68. In all trials, efficacy of fish oil was assessed compared to a placebo. In eleven trials it has been found that fish oil was significantly more effective than placebo in the treatment of RA. However, in one trial, effect of fish oil was similar to the effects of placebo. Stomach upset, diarrhoea and flatulence were reported as adverse effects.
\end{abstract}

Conclusions: Fish oil has consistently demonstrated its clinical benefits in the management of RA.

\section{Introduction}

Rheumatoid arthritis (RA) is an autoimmune disease and is the most common variety of inflammatory arthritis. RA is usually a symmetrical disease affecting both small and large joints. It has lifelong clinical course with intermittent exacerbations and remissions (1). RA is a systematic disease and symptoms like fatigue, anorexia and weight loss are usually associated with the disease (1). Prevalence of RA among general population of UK was found as $1.16 \%$ among females and $0.44 \%$ among males. Female predominance is found in each age group and prevalence increases with age among both males and females (2). Prevalence of RA was found to be varying among different countries and different ethnicities. However, female predominance and increasing incidence with age have remained same (3). Recent research has identified number of factors including obesity, smoking status, blood transfusion, high birth weight and genetic factors as risk factors of RA(4).
A large number of different therapies have been described in the medical literature in relation to the treatment of RA. National Institute of Health and Clinical Excellence (NICE) has evaluated all the available evidence and had prepared a set of guidelines for the management of RA in the United Kingdom (5). According to these guidelines, multidisciplinary team is necessary for the management and specialized care should be sought early. Mainstay of pharmacological treatment includes a combination of disease modifying antirheumatic drugs and analgesics. Patient education, counselling and physiotherapy must go hand in hand with the pharmacological treatment. The NICE does not recommend specific complementary therapy due to the unavailability of strong evidence to support their use. However, due to the chronic nature of the disease and its effects on quality of life, patients and their physicians tend to use alternative methods of treatment for RA(6). 
Fish body oil, a dietary supplement has attracted lot of interest among the patients and rheumatologists as a complementary therapy for RA. Currently it is prescribed by rheumatologists and general practitioners in Sri Lanka. There are several commercial preparations available in Sri Lanka. These oils are extracted by the tissues of fatty fish such as sprat, mackerel, sardine and salmon. Fish body oil is a rich source of omega-3 essential fatty acids which believed to have strong antiinflammatory properties. They act mainly in two ways to relive the symptoms of RA patients. Firstly, they act by reducing the release of pro-inflammatory substances from the white blood cells. Secondly, they provide the necessary constituents for the synthesis of prostaglandins. Prostaglandins can regulate the immune system and fight against the joint inflammation.

Given the popularity of fish body oil, it is important that patients and practitioners have accessible and clear evaluation of the efficacy and safety of these treatments. The purpose of the review is to produce such evidence regarding fish body oil taken orally for the treatment of RA.

\section{Methods}

Eligible publications were sought using a computerized search of the following databases: Allied and Complementary Medicine (1985 to December 2010), EMBASE (1980 to December 2010), Ovid MEDLINE (1950 to December 2010), EBM Reviews ACP Journal Club (1991 to December 2010), EBM Reviews Cochrane Central Register of Controlled Trials ( $3^{\text {rd }}$ Quarter 2010), EBM Reviews Cochrane Database of Systematic Reviews ( $3^{\text {rd }}$ Quarter 2010) and EBM Reviews Database of Abstracts of Reviews of Effects ( $3^{\text {rd }}$ Quarter 2010).

Search terms included key words such as "fish oil", "fish body oil", "omega-3 fatty acids" "alternative medicine", "complementary medicine", "rheumatoid arthritis", "randomized controlled trials", "systematic reviews" and "meta-analysis". Publications published until the end of December 2010 were eligible for inclusion in the review.

\section{Selection Criteria:}

The following criteria were used to select the articles: (a) The study was a randomized controlled trial, (b) conducted on human subjects with RA, (c) route of administration was oral, (d) study was published in English.

Reviewer screened the titles of the selected articles and excluded duplicates and those obviously ineligible. Then reviewed the selected articles to apply the selection criteria. If the information in the abstracts was insufficient to make a decision, full papers were retrieved, and a decision made thereafter. The references of all selected relevant articles including systematic reviews and metaanalysis were manually searched to obtain additional relevant publications. Quality of the RCTs was assessed using JADAD scoring system.

\section{Results}

A total of 48 articles were identified by the computerized search of databases and of them 34 were excluded by examination of their titles. Excluded studies were mainly duplicates, studies on complementary medicine other than fish body oil, studies on rheumatic diseases other than rheumatoid arthritis, study designs other than randomized controlled trials, studies on animals and studies published in languages other than English. Abstracts of the remaining 14 studies and those identified by the screening of references of relevant original and review articles were reviewed. From this process total of 12 articles were included in the review. Identification of relevant studies is detailed in Figure.

Efficacy of Fish body oil in relieving clinical symptoms in RA has been tested in 12 RCTs with sample size ranging from 16 to 68 (Table). In all trials, efficacy of Fish body oil was assessed compared to a placebo. Except in one trial, in all the other 11 trials it has been found that Fish body oil was significantly more effective than placebo in improving one or more clinical symptoms of RA such as pain, morning stiffness, number of tender joints, number of swollen joints and global arthritis activity. In some trials, patients who treated with Fish body oil used significantly less amount of NSAIDs compared to the patients who received placebo. Stomach upset, diarrhoea and flatulence were reported as adverse effects. 


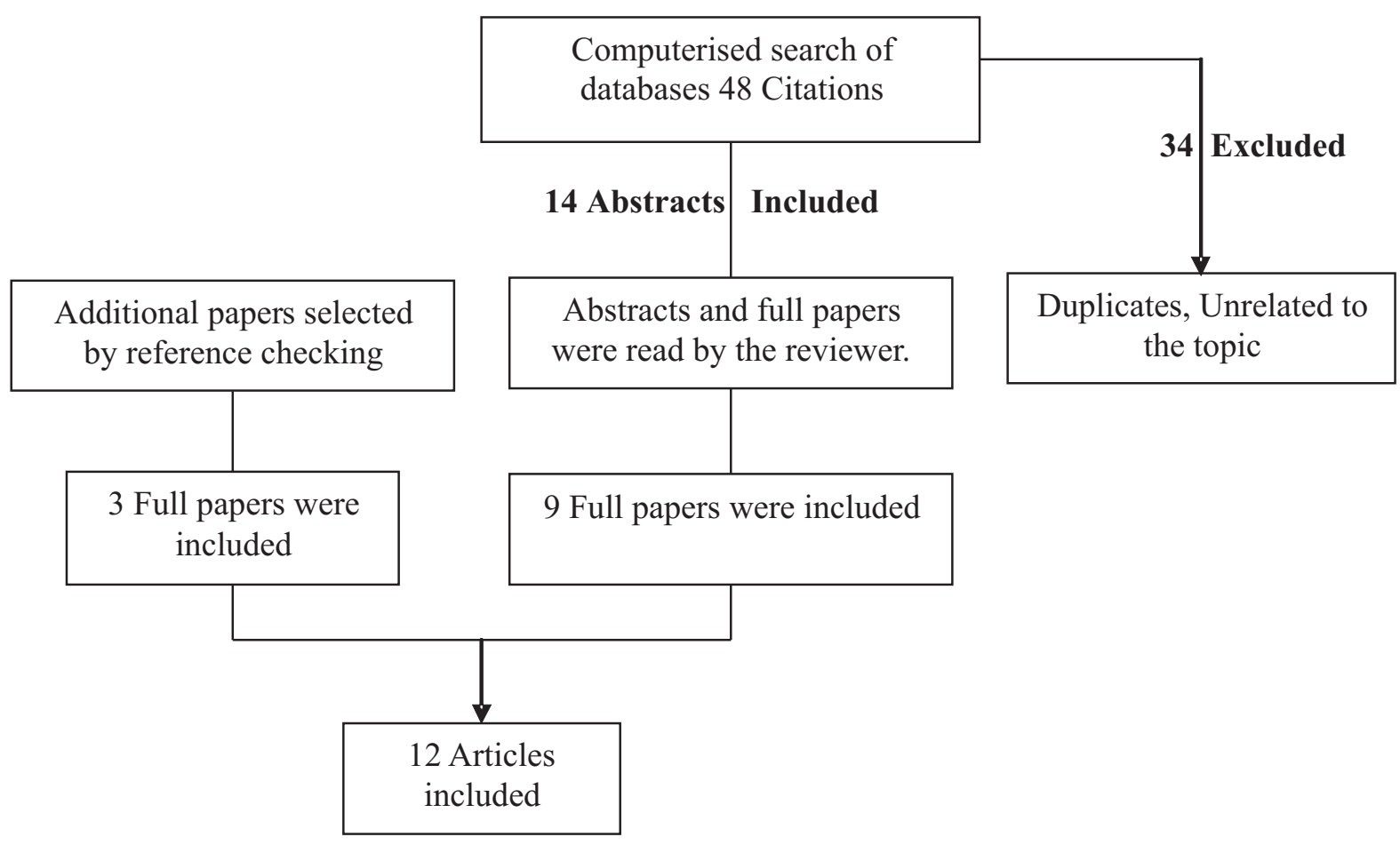

Figure: Process of selecting articles for inclusion in the review

\section{Discussion}

The present review was carried out to determine the efficacy and safety of fish body oil in the management of RA using the available evidence in the form of published RCTs. The review was limited to the articles published in English language and compared the efficacy of fish body oil with a placebo or with a conventional treatment for RA. Few studies which have compared the efficacy of fish body oil with other alternative and complementary medicines were excluded from the review.

The present review found consistent evidence that Fish body oil (7-18) was effective in the management of RA. And it was found to be safe in humans. However, in few studies subjects have reported minor gastro-intestinal side effects such as diarrhoea and flatulence. Assessments of the quality of the trials are important in interpretation of their results. Majority of the reported trials in the current review had relatively high JADAD score values.

Fortin et al (19) reviewed the studies on Fish oil and performed a meta-analysis. According to this metaanalysis, treatment with Fish oil for 3 months significantly reduced the tender joint count $(p=0.001)$ and morning stiffness $(p=0.01)$. They have validated this by a mega-analysis using all the primary data. That analysis also confirmed the results of the meta- analysis. A more recent metaanalysis identified 17 articles investigating the role of fish body oil in patients with RA (20). This metaanalysis confirmed the benefits of fish oil in the reduction of pain, duration of morning stiffness and number of tender or swollen joints in patients with RA. In addition to these, fish oil has significantly reduced the consumption of NSAIDs.

Only RCTs published in English language were considered in this review. This is a major limitation in the present review. We found 3 publications available in other languages. The major concern is a publication bias since researchers are more likely to submit and editors are more likely to publish trials with positive results. However, there was no reliable way to identify the unpublished trials. However, according to this review, fish body oil has consistently demonstrated its clinical efficacy in the management of RA. 


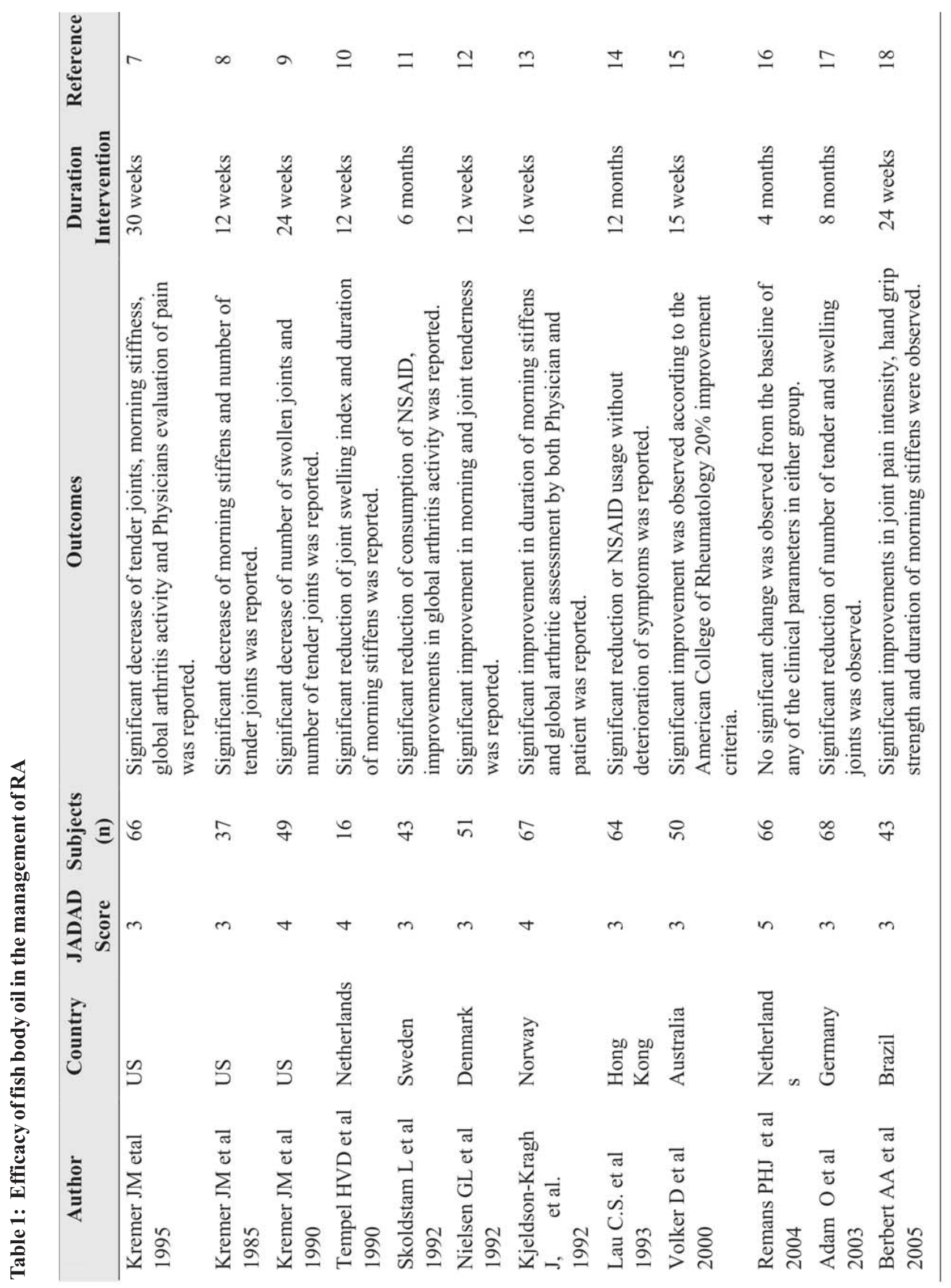




\section{Acknowledgements}

I thank Prof GJ Macfarlane, University of Aberdeen, UK for his guidance in doing systematic reviews and to Ms Sarah Lombordo, Duke University, USA for her assistance in identifying relevant articles.

\section{References}

1. Doherty M, Lanyon P, Ralston SH. Musculoskeletal disorders. Davidson's, principle and practice of Medicine. $20^{\text {th }}$ edition $1101-1103$.

2. Symmons D, Turner G, Webb R, Asten P, et al. The prevalence in Rheumatoid arthritis in United Kingdom: new estimates for a new centaury. Rheumatology, 2002; 41 : 793-800.

3. Guillemin F, Saraux A, Guggenbuhl P, Roux C, et al. Prevalence in rheumatoid arthritis in France: 2001. Ann Rheum Dis, 2005; 64(10): 1427-30.

4. Oliver JE, Silman AJ. What epidemiology has told us about risk factors and aetiopathogenesis in rheumatic disease. Arthritis Research and Therapy, 2009; 11: 223.

5. National Institute for health and Clinical Excellence. Rheumatoid arthritis: The management of rheumatoid arthritis in adults. NICE Clinical Guideline 79. 2009; www.nice.org.uk.

6. Ernst E. Usage of complementary therapies in rheumatology. A systematic review. Clin Rheumatol, 1998; 17: 301-305.

7. Kremer JM, Lawrence DA, Gayle F, Petrillo BS, et al. Effects of high dose fish oil on rheumatoid arthritis after stopping non steroidal anti-inflammatory drugs clinical and immune correlates. Arthritis \& Rheumatism, 1995; 38(8): 1107-14.

8. Kremer JM, Michalek AV, Lininger L, Huyck C, et al. Effects of manipulation of dietary fatty acids on clinical manifestations of rheumatoid arthritis. The Lancet, 1985: 26: $184-87$.

9. Kremer JM, Lawrence DA, Jubiz W, Digiacomo R, et al. Dietary fish oil and olive oil supplementation in patients with rheumatoid arthritis, clinical and immunological effects. Arthritis \& Rheumatism, 1990; 33(6): 810-20.

10. Tempel HVD, Tulleken JE, Limburg PC, Muskiet FAJ, et al. Effects of fish oil supplementation in rheumatoid arthritis. Annals of the Rheumatic Diseases, 1990; 49: 7680.
11. Skoldstam L, Borjesson O, Kjallman A, Seiving B, et al. Effects of six months of fish oil supplementation in stable rheumatoid arthritis. A double blind, controlled study. Scand J Rheumatol, 1992; 21(4): 178-85.

12. Nielsen GL, Faarvang KL, Thomsen BS, et al. The effects of dietary supplementation with n-3 polyunsaturated fatty acids in patients with rheumatoid arthritis: a randomized double blind trial. Eur J Clin Invest, 1992; 22(10); 687-91.

13. Kjeldsen-Kragh J, Lund JA, Riise T, Finnanger B, et al. Dietary omega 3 fatty acids supplementation and naproxen treatment in patients with rheumatoid arthritis. $J$ Rheumatol, 1992;19(10): 1531-36.

14. Lau CS, Morley KD, Belch JJF. Effects of fish oil supplementation on non steroidal anti inflammatory drug requirement in patients with mild rheumatoid arthritis - A double blind placebo controlled study. British Journal of Rheumatology, 1993; 32: 982-89.

15. Volker D, Fitzgerald P, Major G, Garg M. Efficacy of fish oil concentrate in the treatment of rheumatoid arthritis. $J$ Rheumatol, 2000; 27(10): 2343-6.

16. Remans PHJ, Sont JK, Wagenaar LW, Wouters-Wesseling W, et al. Nutrient supplementation with polyunsaturated fatty acids and micronutrients in rheumatoid arthritis: Clinical and biochemical effects. European Journal of Clinical Nutrition, 2004; 58: 839-45.

17. Adam O, Beringer C, Kless T, Lemmen C, et al. Anti inflammatory effects of a low arachidonic acid diet and fish oil in patients with rheumatoid arthritis. Rheumatol Int, 2003; 23: 27-36.

18. Berbert AA, Kondo CRM, Almendra CL, Matsuo T, et al. Supplementation of fish oil and olive oil in patients with rheumatoid arthritis. Nutrition, 2005; 21: 131-36.

19. Fortin PR, Lew RA, Liang MH. Validation of a metaanalysis: the effects of fish oil in rheumatoid arthritis. J Clin Epidemiol, 1995; 48(11): 1379-90.

20. Goldberg RJ, Katz J. A meta analysis of the analgesic effects of omega-3 polyunsaturated fatty acid supplementation for inflammatory joint pain. Pain, 2007; 129: $210-223$. 